I. AMEMIYA, S. OKADA AND Y. OKAZAKI

KODAI MATH. J.

1 (1978), 10l-132

\title{
PRE-RADON MEASURES ON TOPOLOGICAL SPACES
}

\author{
By Ichiro Amemiya, Susumu Okada and Yoshiaki Okazaki
}

\section{§ 1. Introduction.}

There are two directions in the study of the measure theory on arbitrary topological spaces: the theory of Radon measures and the theory of Baire measures. The outline of the developments in these fields is referred to Bourbaki [2], Hirschfeld [8], Schwartz [11] and Varadarajan [13].

The purpose of this paper is to study infinite Borel measures.

Originally, in 1970, the first author has proposed the notion of a pre-Radon measure on a topological space, which is defined as a class of "measures determined by an open base with a smoothness condition" (Amemiya [1]). It seems to be of use for the study of infinite measures, especially Borel measures on a topological space. In this paper, we formulate a pre-Radon measure as a Borel measure (see Definition 2.2) and develop the topics in a survey of Amemiya [1] from a different viewpoint.

Finite pre-Radon measures are said to be $\tau$-smooth Borel measures which have been investigated by many mathematicians. For infinite Borel measures with $\tau$-smoothness, Fremlin [3] recently presented the class of quasi-Radon measures. Our pre-Radon measures are slightly different from quasi-Radon measures.

Main results of this paper are three constructions of pre-Radon measures given in Section 3. The fundamental idea is suggested by Kirk [9]. In Theorem 3.1, we extend a finitely additive set function satisfying some smoothness conditions defined on the ring generated by an open base to a pre-Radon measure. Similarly, in Theorem 3.2 we consider a set function defined on the algebra generated by an open base. In Theorem 3.4, an infinite Baire measure with $\tau$ smoothness on a normal space is extended to a pre-Radon measure. For finite $\tau$-smooth Baire measures, this extension is known (see for example Kirk [9]).

In Section 4, we give the decomposition theorem for $\sigma$-finite pre-Radon measures.

In Section 5, we deal with the restriction of pre-Radon measures. We present the several conditions that the restriction is a pre-Radon measure.

In Section 6, we prove the decomposability of pre-Radon measures. For Radon measures, the decomposability is given in [2, § 1, Proposition 9] and for quasi-Radon measures, Fremlin [2, Theorem 72B].

In section 7 , we give some topological spaces with the $(K)$-property (for the

Recerved October 6, 1976 
definition, see Section 2). We prove if a topological space $X$ is a Borel subset of its Stone-Čech compactification, then $X$ has the (K)-property (Theorem 7.4). In particular, topologically complete spaces and $\sigma$-compact spaces have the $(K)$ property.

In Section 8, we prove that there exists a one-to-one correspondence between pre-Radon measures and smooth linear functionals.

In Section 9, we show the uncountable product of pre-Radon probability measures is uniquely extended to a pre-Radon measure on the product space (Theorem 9.9). In the countable product case, Tortrat [12] has proved the same result, still we show using a Fubini type theorem (Theorem 9.6) for the sake of completeness.

The authors would like to thank Professor T. Ohno for useful information. And they are greatly indebted to Professor H. Sato and Professor Y. Yamasaki for their valuable suggestions for improvements.

\section{§ 2. Preliminaries.}

Let $X$ be a set. A family $U$ of subsets of $X$ is said to be a paving if it satisfies the following conditions:

1) $\phi \in \mathcal{U}$;

2) $\bigcup_{U \in \cup} U=X$;

3) If $U_{1}, U_{2} \in \mathcal{V}$, then $U_{1} \cap U_{2} \in \mathcal{V}$ and $U_{1} \cup U_{2} \in \mathcal{V}$.

We denote by $R[\mathcal{U}]$ the ring generated by a paving $U$.

LemMA 2.1 (Kirk and Crenshaw [10, Proposition 1.2]). Let $F$ be a subset of $X$, then $F$ belongs to $R[\mathcal{U}]$ if and only if there are sets $W_{\imath}, V_{\imath}$ in $\mathcal{U}(\imath=1,2$, $\cdots, n)$ such that the following conditions hold:

1) $V_{i} \subset W_{\imath}(\imath=1,2, \cdots, n)$;

2) $\left(W_{i}-V_{\imath}\right) \cap\left(W_{\jmath}-V_{j}\right)=\emptyset$ for $\imath \neq \jmath$;

3) $F=\bigcup_{\imath=1}^{n}\left(W_{\imath}-V_{\imath}\right)$.

Let $m$ be a non-negative, exiended real valued set function on an algebra $\mathcal{A}$ of subsets of $X$. We say $m$ is $\sigma$-fintte if there exists a countable subfamily $\left\{A_{n} \in \mathcal{A} ; m\left(A_{n}\right)<\infty, n=1,2, \cdots\right\}$ such that $X=\bigcup_{n=1}^{\infty} A_{n}$, and $m$ is semi-finite if $m$ satisfies

$$
m(A)=\sup \{m(B) ; \mathcal{A} \ni B \subset A, m(B)<\infty\}
$$

for every $A$ in $A$. A measure $\mu$ is a non-negative, extended real valued and countably additive set function defined on a $\sigma$-algebra $\mathscr{B}$ such that $\mu(\phi)=0$. 
Let $(X, \mathscr{B}, \mu)$ be a measure space and $A$ be an element in $\mathscr{B}$. We denote by $\mu_{A}$ the measure on the measurable space $(A, A \cap \mathscr{B})$ defined by

$$
\mu_{A}(A \cap B)=\mu(A \cap B)
$$

for every $B$ in $\mathscr{B}$. We call $\mu_{A}$ the restruction of $\mu$ to $A$.

Let $\left\{\left(X, \mathscr{B}_{\lambda}, \mu_{\lambda}\right) ; \lambda \in \Lambda\right\}$ be a family of measure spaces such that $\mu_{\lambda}\left(X_{\lambda}\right)=1$. By $\bigotimes_{\lambda \in A} \mathscr{B}_{\lambda}$, we mean the product $\sigma$-algebra, that is, the smallest $\sigma$-algebra which makes each projection of $\prod_{\lambda \in A} X_{\lambda}$ onto $X_{\lambda}$ measurable. Then there exists a unique probabilty measure $\underset{\lambda \in \Lambda}{\otimes} \mu_{\lambda}$ on $\bigotimes_{\lambda \in \Lambda} \mathcal{B}_{\lambda}$ such that

$$
\left(\otimes_{\lambda \in \Lambda} \mu_{\lambda}\right)(A)=\mu_{\lambda_{1}}\left(A_{\lambda_{1}}\right) \cdots \mu_{\lambda_{n}}\left(A_{\lambda_{n}}\right)
$$

for every set $A$ of the form $A_{\lambda_{1}} \times \cdots \times A_{\lambda_{n}} \times \prod_{\lambda \neq \lambda_{\lambda}} X_{\lambda}$ in $\otimes_{\lambda \in \Lambda} \mathcal{B}_{\lambda}$. This measure $\otimes_{\lambda \in \Lambda} \mu_{\lambda}$ is called the product measure.

Let $X$ be a topological space. By the Borel field $\mathscr{B}(X)$, we mean the minimal $\sigma$-algebra generated by all open subsets of $X$. By $C(X)$, we denote the algebra of all real continuous functions on $X$. The Barre field $\mathscr{B}_{a}(X)$ is the minimal $\sigma$ algebra generated by the family of zero sets

$$
Z(X)=\left\{f^{-1}(0) ; f \in C(X)\right\} .
$$

Now we define pre-Radon measures and Radon measures.

Definition 2.2. Let $X$ be a topological space. A pre-Radon measure $\mu$ is a Borel measure on $\mathscr{B}(X)$ such that:

1) For every $x$ in $X$, there exists an open neighborhood $O$ of $x$ such that $\mu(O)<\infty$;

2) For every net $\left\{O_{\alpha}\right\}$ of open sets increasing to an open subset $O, \lim _{\alpha} \mu\left(O_{\alpha}\right)$ $=\mu(O)$;

3) For every open subset $O$ such that $\mu(O)<\infty$,

$$
\mu(O)=\sup \{\mu(F) ; F \subset O \text { and } F \text { is closed }\} ;
$$

4) For every $A$ in $\mathcal{B}(X)$,

$$
\mu(A)=\inf \{\mu(O) ; O \supset A \text { and } O \text { is open }\} .
$$

We say a Borel measure satisfying 3), 4) a regular Borel measure.

In the same manner as in the proof of Theorem (11.32) of Hewitt and Ross [6], it follows that the above conditions 3$), 4$ ) imply the following 3$)^{\prime}$ :

$3)^{\prime}$ For every $A$ in $\mathscr{B}(X)$ such that $\mu(A)<\infty$,

$$
\mu(A)=\sup \{\mu(F) ; F \subset A \text { and } F \text { is closed }\} .
$$


Consequently, the conditions 3), 4) are equivalent to 3$\left.)^{\prime}, 4\right)$.

Remark 2.3. In general, the above conditions 3$)^{\prime}$ and 4) are not necessarily equivalent. If a Borel measure $\mu$ is $\sigma$-finite and satisfies 4 ). then 3$)^{\prime}$ holds. For infinite Borel measures, the conditions which deduce 4) from 1) and 3$)^{\prime}$ are not known except that $X$ is locally compact and $\sigma$-compact, as far as the authors are concerned. We shall discuss this problem in Appendix.

Remark 2.4. There exists a non-regular Borel measure on a compact space (see Halmos [5, 52, Exercise (10)]). This also gives an example of a Borel measure which is not a pre-Radon measure.

Definition 2.5. Let $X$ be a topological space. A Radon measure $\mu$ is a Borel measure on $\mathscr{B}(X)$ such that

1) For every $x$ in $X$, there exists an open neighborhood $O$ of $x$ such that $\mu(O)<\infty$;

2) For every open set $O$,

$$
\mu(O)=\sup \{\mu(K) ; K \subset O \text { and } K \text { is compact }\} ;
$$

3) For every $A$ in $\mathscr{B}(X)$,

$$
\mu(A)=\inf \{\mu(O) ; O \supset A \text { and } O \text { is open }\} .
$$

Our definition of a Radon measure is different from Bourbaki [2] whose "Radon measure" is a Borel measure satisfying 1) and 2) in Definition 2.5.

It follows that a Radon measure is a pre-Radon measure. Conversely it is easily verified that a pre-Radon measure on a locally compact space is a Radon measure. We say a topological space has the (K)-property if every pre-Radon measure is a Radon measure.

The support of a Borel measure $\mu$ on a topological space $X$ is the set of all points $x$ in $X$ with the property that, for every open set $O$ containing $x, \mu(O)>0$. We denote by $\operatorname{supp} \mu$ the support of $\mu$. We have the following easy consequence.

THEOREM 2.6. Every non-zero pre-Radon measure has the non-empty support.

\section{§3. Construction of pre-Radon measure.} sures.

In this section, we give three methods of constructions of pre-Radon mea-

Firstly, we discuss a set function defined on a ring.

Theorem 3.1. Let $X$ be a topological space, $U$ be a paving generated by an open base of $X$ and $m$ be a non-negative, real valued, finitely additive set function on $R[U]$ such that

1) For any net $\left\{U_{\alpha}\right\}$ of subsets in $U$ increasing to a set $U$ in $U$, 


$$
\lim _{\alpha} m\left(U_{\alpha}\right)=m(U)
$$

2) For every $U$ in $U$,

$$
m(U)=\sup \{m(F) ; U \supset F \in R[\vartheta] \text { and } F \text { is closed }\} .
$$

Then $m$ is uniquely extensible to a pre-Radon measure.

Proof. If two nets $\left\{U_{\alpha}\right\}$ and $\left\{V_{\tau}\right\}$ increase to an open set $O$, then we have

$$
\lim _{\alpha} m\left(U_{\alpha}\right)=\lim _{\tau} m\left(V_{\tau}\right) \text {. }
$$

For every open se: $O$, we put

$$
\lambda(O)=\sup \{m(U) ; O \supset U \in \mathcal{U}\} .
$$

Then it follows that $\lambda$ is a non-negative, monotone and subadditive set function on the family of open subse's of $X$. It can be easily shown that for any net $\left\{O_{\alpha}\right\}$ increasing to $O$,

$$
\lim _{\alpha} \lambda\left(O_{\alpha}\right)=\lambda(O) .
$$

We define a set function $\mu^{*}$ as follows :

$$
\mu^{*}(A)=\inf \{\lambda(O) ; O \supset A \text { and } O \text { is open }\}
$$

for every subset $A$ of $X$. It is evident that $\mu^{*}$ is an outer measure defined on all subsets of $X$. We shall prove that every open subset is $\mu^{*}$-measurable by the way similar to Kirk [9, Lemma 1.12]. Let $O$ be an open subset of $X$ and $A$ be a subset. It is sufficient to show

$$
\mu^{*}(A) \geqq \mu^{*}(A \cap O)+\mu^{*}(A-O) .
$$

We may assume that $\mu^{*}(A)$ is finite. For arbitrary $\varepsilon$ positive, there is an open subset $O_{1}$ containing $A$ such that $\lambda\left(O_{1}\right)<\varepsilon+\mu^{*}(A)$. Let $\left\{U_{\alpha}\right\}$ be a net in $U$ increasing to $O_{1}$ and $V$ be a set in $U$ contained in $O$. By the condition 2), there exists a closed set $F$ in $R[U]$ with $F \supset V$ such that $m(F)+\varepsilon>m(V)$. Then it holds

$$
m\left(U_{\alpha}-F\right)-m\left(U_{\alpha}-V\right) \leqq m(V-F)<\varepsilon,
$$

so that it follows

$$
\varepsilon+\lim _{\alpha} m\left(U_{\alpha}-V\right)<\lim _{\alpha} m\left(U_{\alpha}-F\right)=\lambda\left(O_{1}-F\right) \geqq \mu^{*}(A-O) .
$$

Thus we have

$$
\begin{aligned}
\varepsilon+\mu^{*}(A)>\lambda\left(O_{1}\right) & =\lim _{\alpha} m\left(U_{\alpha}\right) \\
& =\lim _{\alpha}\left(m\left(U_{\alpha} \cap V\right)+m\left(U_{\alpha}-V\right)\right)>\lambda\left(O_{1} \cap V\right)+\mu^{*}(A-O)-\varepsilon .
\end{aligned}
$$


Since $V$ is arbitrary, we have

$$
2 \varepsilon+\mu^{*}(A) \geqq \lambda\left(O_{1} \cap O\right)+\mu^{*}(A-O) \geqq \mu^{*}(A \cap O)+\mu^{*}(A-O),
$$

which shows every open subset is $\mu^{*}$-measurable. So the restriction $\mu$ of $\mu^{*}$ to the Borel field $\mathscr{B}(X)$ is a Borel measure. From the definition of $\mu$, it is obvious that $\mu$ is a pre-Radon measure.

We show $\mu$ is an extension of $m$. By Lemma 2.1, every $A$ in $R[U]$ can be represented as a disjoint union $A=\bigcup_{\imath=1}^{n}\left(W_{i}-V_{\imath}\right)$. Thus we have

$$
m(A)=\sum_{\imath=1}^{n}\left(m\left(W_{\imath}\right)-m\left(V_{\imath}\right)\right)=\sum_{\imath=1}^{n}\left(\mu\left(W_{\imath}\right)-\mu\left(V_{\imath}\right)\right)=\mu(A) .
$$

Finally we shall prove the uniqueness of $\mu$. Let $\nu$ be another pre-Radon measure extending $m$. For any open set $O$, we can find a net $\left\{U_{\alpha}\right\}$ in $\vartheta$ increasing to $O$. Then we have

$$
\mu(O)=\lim _{\alpha} \mu\left(U_{\alpha}\right)=\lim _{\alpha} m\left(U_{\alpha}\right)=\lim _{\alpha} \nu\left(U_{\alpha}\right)=\nu(O) .
$$

By the regularity of $\mu$ and $\nu$, we have

$$
\begin{aligned}
\mu(A) & =\inf \{\mu(O) ; O \supset A \text { and } O \text { is open }\} \\
& =\inf \{\nu(O) ; O \supset A \text { and } O \text { is open }\} \\
& =\nu(A) .
\end{aligned}
$$

This completes the proof.

Secondly we deal with a set function on an algebra.

Theorem 3.2. Let $X$ be a topological space, $U$ be a paving generated by an open base of $X$ and $m$ be a non-negatıve, extended real valued, countably additive set function on the algebra $A[\vartheta]$ generated by $U$. If $m$ satisfies the following conditions:

1) There exists an increasing sequence $\left\{U_{n}\right\}$ in $U$ such that $m\left(U_{n}\right)$ is finite, and $X=\bigcup_{n=1}^{n} U_{n}$;

2) For any net $\left\{U_{\alpha}\right\}$ of subsets in $U$ increasing to a set $U$ in $Q$ such that $m(U)$ is finite,

$$
\lim _{\alpha} m\left(U_{\alpha}\right)=m(U)
$$

3) For every $U$ in $U$ such that $m(U)$ is finte,

$$
m(U)=\sup \{m(F) ; U \supset F \in A[U] \text { and } F \text { is closed }\},
$$

then $m$ is unrquely extended to a pre-Radon measure.

Proof. For every open set $O$, we set 


$$
\lambda(O)=\sup \{m(U) ; O \supset U \in \mathcal{U} \text { and } m(U) \text { is finite }\} .
$$

Furthermore we put

$$
\mu^{*}(A)=\inf \{\lambda(O) ; O \supset A \text { and } O \text { is open }\}
$$

for any subset $A$ of $X$. In the same manner as Theorem 3.1, every open set is $\mu^{*}$-measurable. Moreover, the restriction $\mu$ of $\mu^{*}$ to $\mathscr{B}(X)$ is a pre-Radon measure.

We shall prove that $\mu$ is an extension of $m$. For each $U$ in $U$, the algebra $U \cap A[U]$ is generated by $U \cap U$. In fact the family $\left\{A \subset X ; U \cap A \in A_{U}[U \cap U]\right\}$ is an algebra containing $U$, where $A_{U}[U \cap U]$ denotes the algebra of subsets of $U$ generated by $U \cap U$. So this family contains $A[U]$. By Lemma 2.1, for every $A$ in $A[U]$, we have

$$
U \cap A=\bigcup_{\imath=1}^{n}\left(U \cap W_{\imath}-U \cap V_{\imath}\right) \quad \text { (disjoint union), }
$$

where $W_{\imath}$ and $V_{\imath}$ are in $\mathcal{U}$. Particularly, if $m(U)=\mu(U)$ is finite, we have

$$
\begin{aligned}
m(U \cap A) & =\sum_{\imath=1}^{n}\left(m\left(U \cap W_{\imath}\right)-m\left(U \cap V_{\imath}\right)\right) \\
& =\sum_{i=1}^{n}\left(\mu\left(U \cap W_{\imath}\right)-\mu\left(U \cap V_{\imath}\right)\right) \\
& =\mu(U \cap A) .
\end{aligned}
$$

For every $A$ in $A[U]$, we have

$$
\begin{aligned}
m(A) & =\lim _{n} m\left(U_{n} \cap A\right) \\
& =\lim _{n} \mu\left(U_{n} \cap A\right) \\
& =\mu(A) .
\end{aligned}
$$

Consequently $\mu$ is an extension of $m$.

From the arguments in Theorem 3.1, the uniqueness of $\mu$ is clear. The proof is complete.

Remark 3.3. In Theorem 3.2, if $m$ is totally finite, finitely additive set function on $A[U]$ satisfying the conditions 2 ) and 3 ), then it is easy to verify that $m$ is uniquely extended to a pre-Radon measure.

Lastly we consider a set function defined on the Baire field $\mathscr{B}_{a}(X)$. We recall that a cozero set is the complement of a zero set. We denote by $U(X)$ the family of all cozero sets of $X$.

THEOREM 3.4. Let $X$ be a normal topological space and $m$ be a non-negative extended real valued, finitely additıve set function on $\mathscr{B}_{a}(X)$ satısfyng the follow- 
ing conditions:

1) For any $x$ in $X$, there exists a cozero set $U$ containing $x$ such that $m(U)$ is finite;

2) For any net $\left\{U_{\alpha}\right\}$ of cozero sets increasing to a cozero set $U$,

$$
\lim _{\alpha} m\left(U_{\alpha}\right)=m(U) ;
$$

3) For every Barre set $A$ in $\mathscr{B}_{a}(X)$,

$$
\begin{aligned}
m(A) & =\sup \{m(Z) ; A \supset Z \in Z(X)\} \\
& =\inf \{m(U) ; A \subset U \in U(X)\} .
\end{aligned}
$$

Then $m$ is uniquely extensible to a pre-Radon measure.

Proof. In the same manner as in the proofs of Theorem 3.1 and 3.2, we obiain a pre-Radon measure $\mu$ which coincides with $m$ on $U(X)$. The uniqueness is trivial if $\mu$ is an extension of $m$. We only prove that $\mu$ is an extension of $m$. For every $Z$ in $Z(X)$, we have

$$
\begin{aligned}
\mu(Z) & =\inf \{\mu(O) ; O \supset Z \text { and } O \text { is open }\} \\
& \leqq \inf \{\mu(U) ; Z \subset U \in U(X)\} \\
& =m(Z) .
\end{aligned}
$$

Conversely, since $X$ is normal, for any open set $O$ containing the zero set $Z$, there exists a cozero set $U$ such that $O \supset U \supset Z$. Consequently we have $\mu(Z)=$ $m(Z)$ for every $Z$ in $Z(X)$. Let $A$ be any Baire set in $\mathscr{B}_{a}(X)$. Then we have

$$
\begin{aligned}
\mu(A) & =\inf \{\mu(O) ; O \supset A \text { and } O \text { is open }\} \\
& \leqq \inf \{\mu(U) ; A \subset U \in U(X)\} \\
& =\inf \{m(U) ; A \subset U \in U(X)\} \\
& =m(A) \\
& =\sup \{m(Z) ; A \supset Z \in Z(X)\} \\
& =\sup \{\mu(Z) ; A \supset Z \in Z(X)\} \\
& \leqq \mu(A) .
\end{aligned}
$$

Thus $\mu$ is identical to $m$ on $\mathcal{B}_{a}(X)$. This proves the theorem.

Remark 3.5. We can prove the same result as in Theorem 3.4 even if $m$ is defined on the algebra generated by $Z(X)$. 


\section{$\S 4$. Decomposition theorem.}

LEMMA 4.1. Let $\mu$ be a pre-Radon measure on a regular space $X$. Then there exists a unique Radon measure $\nu$ such that $\nu$ is absolutely contrnuous with respect to $\mu$ and $\nu(K)=\mu(K)$ for every compact subset $K$.

Proof. For any open subset $O$, put

$$
m(O)=\sup \{\mu(K) ; K \subset O \text { and } K \text { is compact }\} .
$$

Then we can easily prove that $\lim _{\alpha} m\left(O_{\alpha}\right)=m(O)$ for every net $\left\{O_{\alpha}\right\}$ of open subsets increasing to an open subset $O$. Let $O_{1}$ and $O_{2}$ be two open subsets, then we have

$$
m\left(O_{1} \cup O_{2}\right) \leqq m\left(O_{1}\right)+m\left(O_{2}\right)
$$

since $\mu$ is a regular Borel measure. Since $X$ is a regular space, we have

$$
m(O)=\sup \{m(W) ; W \subset \bar{W} \subset O \text { and } W \text { is open }\}
$$

for every open set $O$, where $\bar{W}$ is the closure of $W$ in $X$.

We define a set function on the family of all subsets of $X$ as follows:

$$
\nu^{*}(A)=\inf \{m(O) ; O \supset A \text { and } O \text { is open }\} .
$$

Then it follows that $\nu^{*}$ is an outer measure. In the same manner as in the proof of Theorem (11.30) of Hewitt and Ross [6], we can show every Borel subset of $X$ is $\nu^{*}$-measurable. We denote by $\nu$ the restriction of $\nu^{*}$ to $\mathscr{B}(X)$.

For any compact subset $K$, we have $\nu(K)=\mu(K)$. In fact, we have

$$
\begin{aligned}
\nu(K) & =\inf \{m(O) ; O \supset K \text { and } O \text { is open }\} \\
& \leqq \inf \{\mu(O) ; O \supset K \text { and } O \text { is open }\} \\
& =\mu(K) .
\end{aligned}
$$

On the other hand, for any open subset $O$ containing $K$, we have $m(O) \geqq \mu(K)$. Thus we have $\nu(K) \geqq \mu(K)$.

It is obvious that $\nu$ is Radon measure and absolutely continuous with respect to $\mu$. The uniqueness of $\nu$ is obvious from the definition of Radon measure. This completes the proof.

We shall prove the following decomposition theorem.

Theorem 4.2. Let $X$ be a regular space and $\mu$ be a pre-Radon measure on $X$. Then there unquely exist a Radon measure $\nu$ and a pre-Radon measure $\rho$ such that

1) $\mu=\nu+\rho$;

2) $\rho(K)=0$ for every compact subset $K$. 
Furthermore if $\mu$ is $\sigma$-finite, then $\rho$ is singular with repect to $\nu$.

Proof. If we put

$$
U=\{U ; U \text { is open and } \mu(U)<\infty\},
$$

then $\mathcal{U}$ is an open base of $X$. We define a set function $m$ on $R[U]$ by

$$
m(A)=\mu(A)-\nu(A)
$$

for every $A$ in $R[\vartheta]$, where $\nu$ is a Radon measure obtained in Lemma 4.1. By Theorem 3.1, $m$ is uniquely extensible to a pre-Radon measure $\rho$. Then it is clear that $\mu(O)=\nu(O)+\rho(O)$ for every open subset $O$. If we remark that both $\mu$ and $\nu+\rho$ are pre-Radon measures, then we have $\mu=\nu+\rho$. For every compact subset $K$ we have $\rho(K)=\mu(K)-\nu(K)=0$. The uniqueness of the decomposition is obvious.

Assume that $\mu$ is $\sigma$-finite, then $\nu$ is also $\sigma$-finite, which implies for a $\sigma$-compact subset $L, \nu(X-L)=0$. On the other hand we have $\rho(L)=0$. Hence $\rho$ is singular with respect to $\nu$. The theorem is proved.

Remark 4.3. In our original version, we assumed that $\mu$ is $\sigma$-finite. The improvement of the theorem is based on a suggestion of Fremlin (personal communication).

\section{$\S 5$. Restriction of pre-Radon measure.}

In this section we consider the restriction of pre-Radon meaures to subsets.

Let $(X, \mathscr{B}, \mu)$ be a measure space. We denote by $(X, \overline{\mathcal{B}}, \bar{\mu})$ the completion of $(X, \mathscr{B}, \mu)$

LeMma 5.1. Let $\mu$ be a regular Borel measure on a topological space $X$ and $A$ be a subset in $\overline{\mathcal{B}(X)}$. Then the restriction $\bar{\mu}_{A}$ of $\bar{\mu}$ to $A$ is a regular Borel measure on $A$.

Proof. It is obvious from the definition of the completion.

By Lemma 5.1, it is easy to verify the following theorem.

THEOREM 5.2. Let $\mu$ be a pre-Radon measure on a topological space $X$ and $O$ be an open subset of $X$. Then the restriction $\mu_{0}$ of $\mu$ to $O$ is a pre-Rondon measure.

If $\mu$ is semi-finite, then the restriction of $\mu$ to any Borel subset is a preRadon measure. In general we have the following theorem.

Theorem 5.3. Let $\mu$ be a pre-Radon measure on a topological space $X$ and $A$ be a subset in $\overline{\mathcal{B}(X)}$ such that $\bar{\mu}_{A}$ is semi-finte on $(A, A \cap \overline{\mathcal{B}(X)})$. Then $\bar{\mu}_{A}$ is a pre-Radon measure.

Proof. At first, we shall prove in the case that $\bar{\mu}(A)$ is finite. Let $\left\{O_{\alpha}\right\}$ be 
a net of open subsets of $A$ increasing to an open subset $O$ of $A$. Since $A$ belongs to $\overline{\mathscr{B}(X)}$, there exist a set $A_{0}$ in $B(X)$ and a set $N$ such that

$$
A=A_{0} \cup N \text { and } \mu^{*}(N)=0 \text {, }
$$

where $\mu^{*}$ denotes the outer measure induced by $\mu$. There exists an open subset $\tilde{O}_{\alpha}$ of $X$ such that $\tilde{O}_{\alpha} \cap A_{0}=O_{\alpha} \cap A_{0}$ for every $\alpha$. Since $\mu$ is a regular measure, there exists an open subset $\tilde{O}$ of finite measure such that $\tilde{O} \cap A_{0}=O \cap A_{0}$ and $\tilde{O} \subset \cup_{\alpha} O_{\alpha}$. We put

$$
\tilde{U}_{\alpha}=\left(\bigcup_{\beta \leq \alpha} \tilde{O}_{\beta}\right) \cap \tilde{O},
$$

then this net $\left\{\tilde{U}_{\alpha}\right\}$ of subsets of $X$ increases to the open set $\tilde{O}$. Thus we have

$$
\begin{aligned}
\lim _{\alpha} \bar{\mu}_{A}\left(O-O_{\alpha}\right) & =\lim _{\alpha} \mu_{A_{0}}\left(\tilde{O} \cap A_{0}-\tilde{U}_{\alpha} \cap A_{0}\right) \\
& \leqq \lim _{\alpha} \mu\left(\tilde{O}-\tilde{U}_{\alpha}\right)=0 .
\end{aligned}
$$

We consider the general case that $\bar{\mu}_{A}$ is semi-finite. Let $\left\{O_{\alpha}\right\}$ be a net of open subsets of $A$ increasing to an open subset $O$ of $A$. If $\bar{\mu}_{A}(O)$ is finite, then from the first step we have

$$
\begin{aligned}
\bar{\mu}_{A}(O) & =\bar{\mu}_{o}(O)=\lim _{\alpha} \bar{\mu}_{o}\left(O_{\alpha}\right) \\
& =\lim _{\alpha} \bar{\mu}_{A}\left(O_{\alpha}\right) .
\end{aligned}
$$

If $\bar{\mu}_{A}(O)$ is infinite, for any natural number $N$ there exists a set $B$ in $A \cap \overline{\mathscr{B}(X)}$ such that $B \subset O$ and $N<\bar{\mu}_{A}(B)<\infty$. Since the net $\left\{O_{\alpha} \cap B\right\}$ increases to $B$, we have

$$
\begin{aligned}
N<\bar{\mu}_{A}(B) & =\bar{\mu}_{B}(B) \\
& =\lim _{\alpha} \bar{\mu}_{B}\left(O_{\alpha} \cap B\right) \\
& \leqq \lim _{\alpha} \bar{\mu}_{A}\left(O_{\alpha}\right) .
\end{aligned}
$$

Thus we have $\lim _{\alpha} \bar{\mu}_{A}\left(O_{\alpha}\right)=\bar{\mu}_{A}(O)$. By Lemma 5.1, $\bar{\mu}_{A}$ is a pre-Radon measure on $\mathscr{B}(A)$. This completes the proof.

Let $(X, \mathscr{B}, \mu)$ be a measure space and $Y$ be a $\mu$-thick subset of $X$. Then there exists a measure $\mu_{Y}$ on $(Y, \mathscr{B} \cap Y)$ such that

$$
\mu(B \cap Y)=\mu_{Y}(B)
$$

for every set $B$ in $\mathcal{B}$ by Hylmos $[5, \S 17$, Theorem A].

THEOREM 5.4. Let $\mu$ be a pre-Radon measure on a topological space $X$ and $Y$ be a $\mu$-thick subset of $X$. Then $\mu_{Y}$ is a pre-Radon measure on $Y$. 
Proof. Let $\left\{O_{\alpha}\right\}$ be a net of open subsets of $Y$ increasing to an open subset $O$ of $Y$. There exists an open subset $\tilde{O}_{\alpha}$ of $X$ such that $\tilde{O}_{\alpha} \cap Y=O_{\alpha}$. Putting $\tilde{U}_{\alpha}=\bigcup_{\beta \leq \alpha} \tilde{O}_{\beta},\left\{\tilde{U}_{\alpha}\right\}$ is a net of open subsets of $X$ increasing to $\bigcup_{\alpha} \tilde{U}_{\alpha}$ such that $\tilde{U}_{\alpha} \cap Y=O_{\alpha}$. Since $\mu$ is a pre-Radon measure, we have

$$
\begin{aligned}
\mu_{Y}(O) & =\mu_{Y}\left(\left(\bigcup_{\alpha} \tilde{U}_{\alpha}\right) \cap Y\right) \\
& =\mu\left(\bigcup_{\alpha} \tilde{U}_{\alpha}\right) \\
& =\lim _{\alpha} \mu\left(\tilde{U}_{\alpha}\right) \\
& =\lim _{\alpha} \mu_{Y}\left(\tilde{U}_{\alpha} \cap Y\right) \\
& =\lim _{\alpha} \mu_{Y}\left(O_{\alpha}\right) .
\end{aligned}
$$

Since $\mu$ is a regular Borel measure, it is easy to verify that $\mu_{Y}$ is a regular Borel measure. Therefore $\mu_{Y}$ is a pre-Radon measure, which completes the proof.

Let $(X, \mathcal{B}, \mu)$ be a measure space and $A$ be any subset of $X$. We say a set $B$ in $\mathcal{B}$ is a minimal measurable cover of $A$ if $A$ is $\mu_{B}$-thick in $B$, that is, $\left(\mu_{B}\right)_{*}(B-A)=0$, where $\left(\mu_{B}\right)_{*}$ is the inner measure induced by $\mu_{B}$. If $\mu$ is $\sigma$-finite, then there exists a minimal measurable cover of every subset. We define the restriction of $\mu$ to $A$. Since $A$ is $\mu_{B}$-thick in $B,\left(\mu_{B}\right)_{A}$ exists. It is clear that $\left(\mu_{B}\right)_{A}$ is identical to $\left(\mu_{B^{\prime}}\right)_{A}$ for another minimal measurable cover $B^{\prime}$ of $A$. Putting $\mu_{A}=\left(\mu_{B}\right)_{A}$, we call $\mu_{A}$ the restriction of $\mu$ to $A$. tion.

Under the above preparations we have the following final result in this sec-

Theorem 5.5. Let $\mu$ be a pre-Radon measure on a topological space $X$ and $A$ be subset of $X$. If $A$ has a minimal measurable cover $B$ in $\overline{\mathcal{B}(X)}$ such that the restruction $\bar{\mu}_{B}$ of $\bar{\mu}$ is semi-finte on $\left(B, B \cap \overline{\mathcal{B}(X))}\right.$, then the restriction $\mu_{A}$ of $\mu$ to $A$ is a pre-Radon measure.

Proof. It follows from Theorem 5.3 and 5.4.

COROLLARY 5.6. Let $\mu$ be a $\sigma$-finte pre-Radon measure on a topological space $X$. Then for any subset $A$ of $X, \mu_{A}$ is a pre-Radon measure.

Proof. Since $\mu$ is $\sigma$-finite, $A$ has a minimal measurable cover.

Remark 5.7. Fremlin has pointed out the followings:

1) Let $\mu$ be a pre-Radon measure on $X$. If $A$ has a minimal measurable cover, then it holds $\mu_{A}(B)=\inf \left\{\mu^{\prime} C\right) ; C \in \mathscr{S}(X)$ and $\left.C \supset B\right\}$ for every $B$ in $A \cap \overline{\mathscr{B}(X)}$.

2) If $\mu$ is a quasi-Radon measure, then every subset $A$ has a minimal measurable cover. Particularly $\mu$ can be restric ed to $A$ and the restriction " ${ }_{A}$ is a 
quasi-Radon measure on $A$.

\section{§6. Decomposability.}

Let $X$ be a topological space and $\mu$ be a pre-Radon measure on $X$. A subset $A$ of $X$ is called locally negligible if $\mu^{*}(O \cap A)=0$ for every open set $O$ such that $\mu(O)$ is finite, where $\mu^{*}$ denotes the outer measure derived from $\mu$.

For pre-Radon measures, we give the following decomposition theorem which is similar to Bourbaki [2, $\S 1$, Proposition 9].

THEOREM 6.1. Let $\mu$ be a pre-Radon measure on a topological space X. Then there exists a family $\left\{B_{\alpha}\right\}$ of closed sets satrsfying the following:

1) Each $\mu\left(B_{\alpha}\right)$ is finite and supp $\mu_{B_{\alpha}}=B_{\alpha}$;

2) The family $\left\{B_{\alpha}\right\}$ is pairwise disjornt;

3) $X-\bigcup_{\alpha} B_{\alpha}$ is locally negligible;

4) If $A$ is a Borel set of finte measure, the cardinal of $\left\{\alpha ; B_{\alpha} \cap A \neq \emptyset\right\}$ is at most countable and it holds

$$
\mu(A)=\sum_{\alpha} \mu\left(A \cap B_{\alpha}\right) .
$$

Proof. Let $\mathcal{A}$ be the collection of all disjoint families $\left\{C_{\lambda}\right\}$ of closed sets of finite measure satisfying supp $\mu_{C_{\lambda}}=C_{\lambda_{2}}$.

Since the family $\{\phi\}$ satisfies these conditions, the collection $\mathcal{A}$ is non-void. By Zorn's lemma, $\mathcal{A}$ has a maximal family $\left\{B_{\alpha}\right\}$. We shall show the family $\left\{B_{\alpha}\right\}$ satisfies the conditions 1$\left.\left.), 2\right), 3\right)$ and 4$)$. Let $O$ be any open subset of finite measure. If $B_{\alpha} \cap O$ is non-void, then we have

$$
0<\mu_{B_{\alpha}}\left(B_{\alpha} \cap O\right)=\mu\left(B_{\alpha} \cap O\right)<\mu(O)<\infty,
$$

for supp $\mu_{B_{\alpha}}$ equals $B_{\alpha}$. Consequently the cardinal of $\left\{\alpha ; B_{\alpha} \cap O \neq \emptyset\right\}$ is at most countable. Hence $O \cap\left(X-\bigcup_{\alpha} B_{\alpha}\right)$ is a Borel set. Assume $\mu\left(O \cap\left(X-\bigcup_{\alpha} B_{\alpha}\right)\right)$ is positive. Since $\mu$ is regular, there exists a closed subset $F$ of $X$ contained in $O \cap\left(X-\bigcup_{\alpha} B_{\alpha}\right)$ such that $\mu(F)$ is positive. Since $\mu_{F}$ is a pre-Radon measure on $F$ by Theorem 5.3 , the set $B=\operatorname{supp} \mu_{F}$ is closed in $X$ and we have

$$
\mu_{F}(B)=\mu(B)>0 \text {. }
$$

For every $x$ in $B$ and any open neighborhood $V$ of $x$ in $B$, there is an open subset $\tilde{V}$ of $F$ such that $\tilde{V} \cap B=V$. Thus we have

$$
\begin{aligned}
\mu_{B}(V) & =\mu_{F}(V)=\mu_{F}\left(V \cup\left(B^{c} \cap F\right)\right) \\
& \geqq \mu_{F}(\tilde{V})>0,
\end{aligned}
$$

for $\operatorname{supp} \mu_{F}$ is equal to $B$. Hence we obtain 


$$
\operatorname{supp} \mu_{B}=B \text {. }
$$

Consequently the family $\left\{B_{\alpha}\right\} \cup\{B\}$ belongs to $\mathcal{A}$, which contradicts to the maximality of $\left\{B_{\alpha}\right\}$. Therefore we have

$$
\mu\left(O \cap\left(X-\bigcup_{\alpha} B_{\alpha}\right)\right)=0 .
$$

This shows that $X-\bigcup_{\alpha} B$ is locally negligible.

Let $A$ be a Borel set in $\mathscr{B}(X)$ of finite measure. Then it follows that the cardinal of $\left\{\alpha ; A \cap B_{\alpha} \neq \emptyset\right\}$ is at most countable. In fact, there exists an open subset $O$ containing $A$ such that $\mu(O)$ is finite. Hence the set $A \cap\left(X-\cup_{\alpha} B_{\alpha}\right)$ belongs to $\mathscr{B}(X)$ and it holds that

$$
\mu\left(A \cap\left(X-\bigcup_{\alpha} B_{\alpha}\right)\right)=0 .
$$

Thus we have

$$
\mu(A)=\sum_{\alpha}\left(A \cap B_{\alpha}\right)
$$

This completes the proof.

For a set $\left\{a_{\lambda} ; \lambda \in \Lambda\right\}$ of non-negative numbers, we define the sum of $\left\{a_{\lambda}\right.$; $\lambda \in \Lambda\}$ by

$$
\sum_{\lambda \in \Lambda} a_{\lambda}=\sup \left\{\sum_{\lambda \in \Lambda_{0}} a_{\lambda} ; \Lambda_{0} \text { is a finite subset of } \Lambda\right\} .
$$

In the semi-finite case the following corollary holds.

COROLlary 6.2. Let $\mu$ be a semi-finite pre-Radon measure on a topological space $X$. Then for the family $\left\{B_{\alpha}\right\}$ obtamed in Theorem 6.1 we have

$4)^{\prime}$ For every $A$ in $\mathcal{B}(X)$;

$$
\mu(A)=\sum_{\alpha} \mu\left(A \cap B_{\alpha}\right) ;
$$

5) $\mu_{*}\left(X-\bigcup_{\alpha} B_{\alpha}\right)=0$,

where $\mu_{*}$ denotes the inner measure defined from $\mu$.

Proof. Since $\mu$ is semi-finite, we have

$$
\begin{aligned}
\mu(A) & =\sup \{\mu(B) ; B \subset A \text { and } \mu(B) \text { is finite }\} \\
& =\sup \left\{\sum_{\alpha} \mu\left(B \cap B_{\alpha}\right) ; B \subset A \text { and } \mu(B) \text { is finite }\right\} \\
& \leqq \sum_{\alpha} \mu\left(A \cap B_{\alpha}\right) \leqq \mu(A),
\end{aligned}
$$

which shows 4$)^{\prime}$.

Let $C$ be any Borel set in $\mathscr{B}(X)$ contained in $X-\bigcup_{\alpha} B_{\alpha}$. For any Borel set 
$B$ of finite measure contained in $X-\bigcup_{\alpha} B_{\alpha}$ we have $\mu(B)=0$ by Theorem 6.1. Thus we have

$$
\begin{aligned}
\mu(C) & =\sup \{\mu(B) ; B \subset C \text { and } \mu(B) \text { is finite }\} \\
& \leqq \sup \left\{\mu(B) ; B \subset X-\cup_{\alpha} B_{\alpha} \text { and } \mu(B) \text { is finite }\right\} \\
& =0 .
\end{aligned}
$$

\section{§ 7. $(\mathrm{K})$-property.}

Let $u$ be a pre-Radon measure on a topological space $X$ and $f$ be a continuous mapping of $X$ into another topological space $Y$. We denote by $f(\mu)$ the image measure of $\mu$ defined by

$$
f(\mu)(A)=\mu\left(f^{-1}(A)\right)
$$

for every Borel set $A$ in $\mathscr{B}(Y)$. In general, it is not true that the image measure $f(\mu)$ is a pre-Radon measure. But the following theorem holds.

THEOREM 7.1. Let $\mu$ be a pre-Radon measure on a topological space $X$ and $f$ be a continuous mapping of $X$ into a regular space $Y$. If we put

$$
\begin{aligned}
Y_{0}= & \{y \in Y \text {; there exists an open neighborhood } U \text { of } y \\
& \text { such that } \left.\mu\left(f^{-1}(U)\right)<\infty\right\},
\end{aligned}
$$

then there uniquely exists a pre-Radon measure $\nu$ on $Y_{0}$ such that

$$
\nu(O)=\mu\left(f^{-1}(O)\right)
$$

for every open subset $O$ of $Y_{0}$.

Proof. We remark that $Y_{0}$ is an open subset of $Y$. If we put

$$
\mathcal{U}=\left\{U \subset Y_{0} ; U \text { is open in } Y_{0} \text { and } \mu\left(f^{-1}(U)\right) \text { is finite }\right\} \text {, }
$$

then $U$ is an open base of $Y_{0}$. If we define a set function $m$ on $R[U]$ by

$$
m(A)=\mu\left(f^{-1}(A)\right)
$$

for every $A$ in $R[\vartheta]$, then for any net $\left\{U_{\alpha}\right\}$ of subsets in $U$ increasing to $U$ in $U$ it follows that

$$
\begin{aligned}
\lim _{\alpha} m\left(U_{\alpha}\right) & =\lim _{\alpha} \mu\left(f^{-1}\left(U_{\alpha}\right)\right) \\
& =\mu\left(f^{-1}(U)\right)=m(U) .
\end{aligned}
$$

Since $Y$ is regular, we have

$$
m(U)=\sup \{m(V) ; V \subset \bar{V} \subset U \text { and } V \text { is open }\}
$$


for every $U$ in $U$, where $\bar{V}$ is the closure of $V$ in $Y$. By Theorem 3.1, there exists a pre-Radon measure $\nu$ on $Y_{0}$ extending $m$. For each open subset $O$ of $Y$ there exists a net $\left\{U_{\alpha}\right\}$ in $U$ increasing to $O$. Thus we have

$$
\begin{aligned}
\nu(O) & =\sup _{\alpha} \nu\left(U_{\alpha}\right) \\
& =\sup _{\alpha} m\left(U_{\alpha}\right) \\
& =\sup _{\alpha} \mu\left(f^{-1}\left(U_{\alpha}\right)\right) \\
& =\mu\left(f^{-1}(O)\right) .
\end{aligned}
$$

The uniqueness of $\nu$ is clear, which completes the proof.

COROLlary 7.2. In the above theorem, if $\mu$ is finite, then $Y_{0}$ equals $Y$ and $f(\mu)$ is a pre-Radon measure. Therefore $\nu$ is identical with $f(\mu)$.

If $X$ is a Borel subset of $Y$, then the restriction of $\nu$ to $\mathscr{B}(X)$ is identical to $\mu$ on $\mathscr{B}(X)$.

LEMMA 7.3. Let $X$ be a Borel subset of a regular space $Y, \mu$ be a pre-Radon measure on $X$ and $\nu$ be the pre-Radon measure obtained in Theorem 7.1. Then the restriction of $\nu$ to $\mathscr{B}(X)$ is equal to $\mu$ on $\mathscr{B}(X)$.

Proof. Let $A$ be any Borel set in $\mathscr{B}(X)$ and $W$ be an open subset of $X$ containing $A$. Then there exists an open subset $\widetilde{W}$ of $Y_{0}$ such that $W=\widetilde{W} \cap X$. Hence we have

$$
\begin{aligned}
\mu(W)=\nu(\widetilde{W}) & \geqq \inf \left\{\nu(\tilde{O}) ; \tilde{O} \supset A \text { and } \tilde{O} \text { is open in } Y_{0}\right\} \\
& =\nu(A)
\end{aligned}
$$

by Theorem 7.1 . Since $\mu$ is regular, we have

$$
\begin{aligned}
\mu(A) & =\inf \{\nu(W) ; W \supset A \text { and } W \text { is open in } X\} \\
& \geqq \nu(A) .
\end{aligned}
$$

Conversely, we have

$$
\begin{aligned}
\nu(A) & =\inf \left\{\nu(\tilde{O}) ; \tilde{O} \supset A \text { and } \tilde{O} \text { is open in } Y_{0}\right\} \\
& =\inf \left\{\mu(\tilde{O} \cap X) ; \tilde{O} \supset A \text { and } \tilde{O} \text { is open in } Y_{0}\right\} \\
& \geqq \inf \{\mu(O) ; O \supset A \text { and } O \text { is open in } X\} \\
& =\mu(A) .
\end{aligned}
$$

This proves the lemma.

We present a sufficient condition under which a topological space has the 
(K)-property.

THEOREM 7.4. Let $X$ be a completely regular Hausdorff space such that $X$ is a Borel subset of 2 ts Stone-Čech compactification $\beta X$. Then every pre-Radon measure $\mu$ on $X$ is a Radon measure, that is, $X$ has the (K)-property.

Proof. Let $c$ be the natural embedding of $X$ into $\beta X,(\beta X)_{0}$ be the open subset of $\beta X$ obtained in Theorem 7.1 and $\nu$ be the pre-Radon measure on $(\beta X)_{0}$ in Theorem 7.1. Since $(\beta X)_{0}$ is locally compact, $\nu$ is a Radon measure. If we remark that the restriction of the Radon measure $\nu$ to the Borel set $X$ is a Radon measure on $X, \mu$ is a Radon measure by Lemma 7.3. The proof is complete.

We recall that a completely regular Hausdorff space is topologically complete if it is a $G_{\tilde{o}}$-subset of its Stone-Čech compactification.

COROLLARY 7.5. Every topologically complete space has the (K)-property. Partıcularly, a complete metruc space has the (K)-property.

COROLlaRY 7.6. Every completely regular Haussdorff, o-compact space has the (K)-property.

\section{§ 8. Smooth linear functional.}

In this section, we show that there is a one-to-one correspondence between pre-Radon measures and smooth linear functionals.

Let $C(X)$ be the Riesz space of all real continuous functions on a completely regular Hausdorff space $X$. A Riesz subspace $J$ of $C(X)$ is said to be orderdense if for every $x$ in $X$, there exists $f$ in $J$ such that $f(x) \neq 0$. A positive linear functional $\Phi$ on a Riesz subspace $J$ of $C(X)$ is called smooth if for every net $\left\{f_{\alpha}\right\}$ in $J$ decreasing to 0 ,

$$
\lim _{\alpha} \Phi\left(f_{\alpha}\right)=0
$$

THEOREM 8.1. I et $\mu$ be a pre-Radon measure on a completely regular Hausdorff space $X$ and $J_{\mu}$ be the Riesz subspace of all $\mu$-integrable continuous functions. Then $J_{\mu}$ is an order-dense ideal and the functional $\Phi_{\mu}$ on $J_{\mu}$ defined by

is a smooth linear functional.

$$
\Phi_{\mu}(f)=\int_{X} f d \mu
$$

Proof. For every $x$ in $X$, there exists an open neighborhood $U$ of $x$ such that $\mu(U)$ is finite. There exists $f$ in $C(X)$ such that

1) $0 \leqq f \leqq 1$;

2) $f(x)=1$;

3) $f=0$ on $U^{c}$, 
where $U^{c}$ means the complement of $U$. Since it holds

$$
\Phi_{\mu}(f)=\int_{X} f d \mu \leqq \mu(U)<\infty,
$$

$f$ belongs to $J_{\mu}$, which shows $J_{\mu}$ is an order-dense ideal.

Let $\left\{f_{\alpha}\right\}$ be a net of non-negative functions in $J_{\mu}$ increasing to $f$ in $J_{\mu^{*}}$. Since $f$ is non-negative, we have

$$
\begin{aligned}
\Phi_{\mu}(f) & =\int_{X} f d \mu=\int_{n}\left\{x ; f(x)>\frac{1}{n}\right\} \\
& =\lim _{n \rightarrow \infty} \int_{\left\{x ; f(x)>-\frac{1}{n}\right\}} f d \mu .
\end{aligned}
$$

For any positive $\varepsilon$, there exists $n$ such that

$$
\Phi_{\mu}(f)<\int_{\left\{x ; f(x)>\frac{1}{n}\right\}} f d \mu+\varepsilon .
$$

Since the sequence $\{f \wedge m\}$ converges to $f$, there exists $m$ such that

$$
\int_{\left\{x ; f(x)>\frac{1}{n}\right\}} f d \mu<\int_{\left\{x ; f(x)>\frac{1}{n}\right\}} f \wedge m d \mu+\varepsilon .
$$

If we remark that $\mu(\{x ; f(x)>1 / n\})$ is finite and $f \wedge m$ is bounded, in the same manner as in the proof of Theorem 24 of Varadrajan [13, Part I] we obtain

$$
\begin{aligned}
\int_{\left\{x ; f(x)>\frac{1}{n}\right\}} f \wedge m d \mu & =\lim _{\alpha} \int_{\left\{x ; f(x)>\frac{1}{n}\right\}} f_{\alpha} \wedge m d \mu \\
& =\lim _{\alpha} \int_{X} f_{\alpha} d \mu .
\end{aligned}
$$

Thus we have

$$
\Phi_{\mu}(f)=\lim _{\alpha} \Phi_{\mu}\left(f_{\alpha}\right)
$$

Let $\left\{f_{\alpha}\right\}$ be a net in $J_{\mu}$ decreasing to 0 . If we fix $\alpha_{0}$, the net $\left\{f_{\alpha_{0}}-f_{\alpha}\right\}$ increases to $f_{\alpha_{0}}$. Then we have

$$
\lim _{\alpha} \Phi_{\mu}\left(f_{\alpha_{0}}-f_{\alpha}\right)=\Phi_{\mu}\left(f_{\alpha_{0}}\right)
$$

Hence we have

$$
\lim _{\alpha} \Phi_{\mu}\left(f_{\alpha}\right)=0
$$

This completes the proof.

The idea of the proof of the following theorem is essentially due to Fremlin [3], Hewitt and Ross [6] and Kirk [9].

Proposition 8.2. Let $J$ be an order-dense ideal of $C(X)$ and $\Phi$ be a non- 
negative smooth linear functional on $J$. Then there exists a unique pre-Radon measure $\mu$ such that $J$ is contained in $J_{\mu}$ and

$$
\Phi(f)=\int_{X} f d \mu
$$

for every $f$ in $J$.

Proof. Let $M^{+}$be the set of functions $\left\{\lim _{\alpha} f_{\alpha} ;\left\{f_{\alpha}\right\}\right.$ is an increasing net, each $f_{\alpha}$ belongs to $\left.J^{+}\right\}$, where $J^{+}$is the subspace of all non-negative functions in $J$. We define a functional $\bar{\Phi}$ on $M^{+}$, by

$$
\bar{\Phi}\left(\lim _{\alpha} f_{\alpha}\right)=\lim _{\alpha} \Phi\left(f_{\alpha}\right) .
$$

This definition of $\bar{\Phi}$ is well-defined, in fact, if $\lim _{\alpha} f_{\alpha}=\lim _{\lambda} f_{\lambda}^{\prime}$ in $M^{+}$it follows that

$$
\lim _{\alpha} \Phi\left(f_{\alpha}\right)=\lim _{\lambda} \Phi\left(f_{\lambda}^{\prime}\right) .
$$

From the definition, we have

$$
\bar{\Phi}(c g)=c \bar{\Phi}(g)
$$

for every $g$ in $M^{+}$and any positive number $c$.

For every net $\left\{g_{\alpha}\right\}$ in $M^{+}$increasing to $g$ in $M^{+}$, we obtain

$$
\lim _{\alpha} \bar{\Phi}\left(g_{\alpha}\right)=\bar{\Phi}(g)
$$

in the same manner as in the proof of Theorem 11.13 of Hewitt and Ross [6]. Therefore we have

for $g_{1}, g_{2}$ in $M^{+}$.

$$
\bar{\Phi}\left(g_{1}+g_{2}\right)=\bar{\Phi}\left(g_{1}\right)+\bar{\Phi}\left(g_{2}\right)
$$

Since $J$ is an order-dense ideal and $X$ is completely regular, the characteristic function $\chi_{0}$ of an open subset $O$ of $X$ belongs to $M^{+}$. We put

$$
m(O)=\bar{\Phi}\left(\chi_{0}\right)
$$

for every open subset $O$. Then for any net $\left\{O_{\alpha}\right\}$ of open subsets increasing to an open subset $O$, we have

$$
\lim _{\alpha} m\left(O_{\alpha}\right)=m(O) .
$$

Moreover for open subsets $O_{1}$ and $O_{2}$, we obtain

$$
m\left(O_{1} \cup O_{2}\right) \leqq m\left(O_{1}\right)+m\left(O_{2}\right)
$$

in the same manner as in the proof of Lemma 1.10 of Kirk [9].

If we set

$$
\mu^{*}(A)=\inf \{m(O) ; O \supset A \text { and } O \text { is open }\}
$$


for every subset $A$ of $X$, then $\mu^{*}$ is an outer measure. Since $X$ is a regular space, it holds

$$
m(O)=\sup \{m(W) ; W \subset \bar{W} \subset O \text { and } W \text { is open }\}
$$

for every open subset $O$. Hence it follows that every Borel subset is $\mu^{*}$-measurable (see for example, Hewitt and Ross [6, Theorem (11.30)]). If we denote by $\mu$ the restriction of $\mu^{*}$ to the Borel field $\mathcal{B}(X), \mu$ is a pre-Radon measure.

We show it holds that

$$
\Phi(f)=\int_{X} f d \mu
$$

for each $f$ in $J^{+}$. In the same manner as in the proof (a) of Lemma $71 \mathrm{~F}$ of Fremlin [3], we have

$$
\Phi(f) \geqq \int f d \mu
$$

for every $f$ in $J^{+}$.

In order to prove the converse, we slightly modify the proof (b) of Lemma $71 \mathrm{~F}$ of Fremlin [3]. For any $f$ in $J^{+}$, we put

$$
f_{n}=f \wedge 2^{n}-f \wedge 2^{-n},
$$

then the sequence $\left\{f-f_{n} ; n=1,2, \cdots\right\}$ in $J^{+}$decreases to 0 . Therefore for an $J$ positive number $\varepsilon$, there exists $n$ such that

If we set

$$
\Phi(f) \leqq \Phi\left(f_{n}\right)+\varepsilon .
$$

$$
H=\left\{x: f(x) \geqq 2^{-n}\right\},
$$

$\mu(H)$ is finite. There exists a positive number $c$ such that

$$
\mu(U)-\mu(H)<\varepsilon / 2^{n},
$$

where $U=\left\{x ; f(x)>2^{-n}-c\right\}$. By Varadarajan [13, Part I, Theorem 10], there exists $g$ in $J^{+}$such that

1) $0 \leqq g \leqq 1$;

2) $g=1$ on $H$;

3) $g=0$ on $U^{c}$.

Then we have

$$
0<\Phi(g)-\mu(H)<\varepsilon / 2^{n}
$$

Thus we obtain

$$
\begin{aligned}
\Phi(f) & \leqq \Phi\left(f_{n}\right)+\varepsilon \\
& =\Phi\left(f_{n}\right)+\bar{\Phi}\left(2^{n} g\right)-\bar{\Phi}\left(2^{n} g\right)+\varepsilon \\
& =2^{n} \bar{\Phi}(g)-\bar{\Phi}\left(2^{n} g-f_{n}\right)+\varepsilon
\end{aligned}
$$




$$
\begin{aligned}
& \leqq 2^{n} \Phi(g)-\int_{X}\left(2^{n} g-f_{n}\right) d \mu+\varepsilon \\
& \leqq 2^{n} \mu(H)+\varepsilon-\int_{X} 2^{n} g d \mu+\int_{X} f_{n} d \mu+\varepsilon \\
& \leqq 2^{n} \mu(H)-2^{n} \mu(H)+\int_{X} f_{n} d \mu+2 \varepsilon \\
& \leqq \int_{X} f d \mu+2 \varepsilon .
\end{aligned}
$$

Hence we have

$$
\Phi(f)=\int_{X} f d \mu
$$

for every $f$ in $J^{+}$.

Lastly we prove the uniqueness. Let $O$ be any open subset of $X$ and $\left\{f_{\alpha}\right\}$ be any net in $J^{+}$increasing to $\chi_{0}$, we have

$$
\mu(O)=\lim _{\alpha} \int_{X} f_{\alpha} d \mu .
$$

In fact, for $0<\delta<1$, putting

$$
U_{\alpha}=\left\{x ; f_{\alpha}(x)>\delta\right\},
$$

$\left\{U_{\alpha}\right\}$ increases to $O$. Since it follows

$$
\lim _{\alpha} \mu\left(U_{\alpha}\right)=\mu(O),
$$

we have

$$
\int_{X} f_{\alpha} d \mu \geqq \int_{U_{\alpha}} f_{\alpha} d \mu \geqq \delta \mu\left(U_{\alpha}\right)
$$

Thus we have

Since $\delta$ is arbitrary, we have $\frac{1}{\delta} \lim _{\alpha} \int_{X} f_{\alpha} d \mu \geqq \mu(O)$.

$$
\mu(O) \geqq \lim _{\alpha} \int_{X} f_{\alpha} d \mu \geqq \mu(O) .
$$

Let $\nu$ be another pre-Radon measure satisfying

$$
\Phi(f)=\int_{X} f d \mu=\int_{X} f d \nu
$$

for every $f$ in $J^{+}$. By the preceeding argument, we have

$$
\mu(O)=\nu(O)
$$

for each open subset $O$. Since $\mu$ and $\nu$ are regular measures, $\nu$ is identical with $\mu$. The theorem is proved. 


\section{§ 9. Product measure.}

The purpose of this section is to study the product of pre-Radon measures.

The proof of the following lemma is easy.

Lemma 9.1. Let $X$ be a topological space, $U$ be a paving generated by an open base containing $X$ and $m$ be a non-negative, totally finte real valued finitely additive set function on $R[U]$ such that

1) For any net $\left\{U_{\alpha}\right\}$ of subsets in $U$ increasing to $X$,

$$
\lim _{\alpha} m\left(U_{\alpha}\right)=m(X)
$$

2) For every $U$ in $U$,

$$
m(U)=\sup \{m(F) ; U \supset F \in R[\mathcal{U}] \text { and } F \text { is closed }\} .
$$

Then we have for any net $\left\{U_{\alpha}\right\}$ of subsets in $U$ increasing to a set $U$ in $U$,

$$
\lim _{\alpha} m\left(U_{\alpha}\right)=m(U) .
$$

To begin with, we investigate the finite product case.

THEOREM 9.2. Let $\mu, \nu$ be totally fintte pre-Radon measures on topological spaces $X, Y$ respectively. Then the product measure $\rho=\mu \otimes \nu$ on $(X \times Y, \mathscr{B}(X) \otimes$ $\mathscr{B}(Y))$ is uniquely extensible to a pre-Radon measure on the product space $X \times Y$.

Proof. Let $U$ be the paving generated by

$$
\{U \times V ; U(\text { resp. } V) \text { is open in } X(\text { resp. } Y)\}
$$

and $\left\{W_{\alpha}\right\}$ be a net of subsets in $\mathcal{U}$ increasing to $X \times Y$. If $\left\{U_{\gamma} \times V_{\gamma}\right\}$ is the collection of open subsets of $X \times Y$ such that each $U_{\gamma} \times V_{\gamma}$ is contained in $W_{\boldsymbol{\alpha}}$ for some $\alpha$, we have $\bigcup_{\gamma}\left(U_{\gamma} \times V_{\gamma}\right)=X \times Y$. For every $x$ in $X$, the family $\left\{V_{\gamma} ; x\right.$ belongs to $\left.U_{\gamma}\right\}$ covers $Y$. Since $\nu$ is a pre-Radon measure, for any positive number $\varepsilon$, there exists $\left\{\gamma_{1}^{x}, \gamma_{2}^{x}, \cdots, \gamma_{n(x)}^{x}\right\}$ such that

$$
\nu\left(\bigcup_{i=1}^{n(x)} V_{\gamma_{\imath} x}\right)>\nu(Y)-\varepsilon \text {. }
$$

We set $U(x)=\bigcap_{\imath=1}^{n(x)} U_{\gamma_{i} x}$. Since $\mu$ is a pre-Radon measure, there exists $\left\{x_{1}, \cdots, x_{k}\right\}$ such that

$$
\mu\left(\bigcup_{j=1}^{k} U\left(x_{\jmath}\right)\right)>\mu(X)-\varepsilon .
$$

From the definition of product measures, it follows 


$$
\begin{aligned}
\rho\left(\bigcup_{j=1}^{k} \bigcup_{i=1}^{n\left(x_{j}\right)}\left(U\left(x_{j}\right) \times V_{\gamma_{i} x_{\jmath}}\right)\right)=\rho\left(\bigcup_{j=1}^{k}\left[\left(U\left(x_{\jmath}\right)-\bigcup_{p=1}^{j-1} U\left(x_{p}\right)\right) \times \bigcup_{i=1}^{n\left(x_{j}\right)} V_{\left.\gamma_{i} x_{\jmath}\right]}\right)\right. \\
=\sum_{j=1}^{k} \rho\left(\left(U\left(x_{\jmath}\right)-\bigcup_{p=1}^{j-1} U\left(x_{p}\right)\right) \times \bigcup_{i=1}^{n\left(x_{j}\right)} V_{\gamma_{i} x_{j}}\right) \\
>\sum_{j=1}^{k} \mu\left(U\left(x_{\jmath}\right)-\bigcup_{p=1}^{j-1} U\left(x_{p}\right)\right)(\nu(Y)-\varepsilon) \\
=\mu\left(\bigcup_{j=1}^{k} U\left(x_{\jmath}\right)\right)(\nu(Y)-\varepsilon)>(\mu(X)-\varepsilon)(\nu(Y)-\varepsilon) .
\end{aligned}
$$

Since $\left\{W_{\alpha}\right\}$ is directed, there exists $\alpha_{0}$ such that

$$
W_{\alpha_{0}} \supset \bigcup_{\jmath=1}^{k} \bigcup_{\imath=1}^{n(x j)} U\left(x_{\jmath}\right) \times V_{r_{\imath} x_{\jmath}} .
$$

Therefore we have

$$
\lim _{\alpha} \rho\left(W_{\alpha}\right)=\rho(X \times Y) .
$$

By Lemma 9.1 and Theorem 3.2, the restriction $\rho_{0}$ of $\rho$ to $R[U]=A[U]$ is uniquely extended to a pre-Radon measure $\bar{\rho}_{0}$ on $X \times Y$. By Halmos $[5, \S 13$, Theorem A], $\rho$ coincides with $\bar{\rho}_{0}$ on the product $\sigma$-algebra $\mathscr{B}(X) \otimes \mathscr{B}(Y)$. This completes the proof.

Now we argue $\sigma$-finite product measures on finite product spaces in the following theorem.

THEOREM 9.3. Let $\mu, \nu$ be $\sigma$-finite pre-Radon measures on topological spaces $X, Y$ respectively. Then the product measure $\rho=\mu \otimes \nu$ on $(X \times Y, \mathscr{B}(X) \otimes \mathcal{B}(Y))$ is uniquely extensible to a pre-Radon measure on $X \times Y$.

Proof. Let $U$ be the paving generated by

$$
\begin{gathered}
\{U \times V ; U(\text { resp. } V) \text { is open in } X(\text { resp. } Y) \text { and } \\
\mu(U)<\infty, \nu(V)<\infty\}
\end{gathered}
$$

and $\left\{W_{\alpha}\right\}$ be a net in $\mathcal{U}$ increasing to $W$ in $\mathcal{U}$. If we write $W=\bigcup_{\imath=1}^{n}\left(U_{\imath} \times V_{\imath}\right)$, we have $W \subset U_{0} \times V_{0}$, where $U_{0}=\bigcup_{\imath=1}^{n} U_{\imath}$ and $V_{0}=\bigcup_{\imath=1}^{n} V_{\imath}$. By Theorem $9.2 \mu_{U_{0}} \otimes \nu_{V_{0}}$ is extensible to a pre-Radon measure on $U_{0} \times V_{0}$. Thus we have

$$
\begin{aligned}
\lim _{\alpha}(\mu \otimes \nu)\left(W_{\alpha}\right) & =\lim _{\alpha}\left(\mu_{U_{0}} \otimes \nu_{V_{0}}\right)\left(W_{\alpha}\right) \\
& =\left(\mu_{U_{0}} \otimes \nu_{V_{0}}\right)(W) \\
& =\mu \otimes \nu(W) .
\end{aligned}
$$


By Theorem 3.2 the restriction $\rho_{0}$ of $\rho$ to $A[\vartheta]$ is uniquely extensible to a preRadon measure $\bar{\rho}_{0}$. Since $\rho$ is $\sigma$-finite, $\bar{\rho}_{0}$ is an extension of $\rho$, which proves the theorem.

Next we investigate a Fubini type theorem.

LEMMA 9.4. Let $\mu$ be a semi-finte pre-Radon measure on a topological space $X$ and $M^{+}(X)$ be the set of all non-negative, extended real valued lower semicontinuous functions on $X$. If a net $\left\{f_{\alpha}\right\}$ in $M^{+}(X)$ increases to $f$ in $M^{+}(X)$, then we have

$$
\lim _{\alpha} \int_{X} f_{\alpha} d \mu=\int_{X} f d \mu
$$

Proof. Firstly we prove the case that $f$ is a simple function $\sum_{i=1}^{n} a_{i} \chi_{E_{i}}$, where $\left\{E_{i}\right\}$ is disjoint. Since $\mu_{E_{\imath}}$ is a pre-Radon measure (Theorem 5.3), by the way similar to the proof of Theorem 8.2 we have

$$
\left.\lim _{\alpha} \int_{E_{\imath}} f_{\alpha}\right|_{E_{i}} d \mu_{E_{i}}=a_{i} \mu\left(E_{\imath}\right) .
$$

Thus we obtain

$$
\begin{aligned}
\lim _{\alpha} \int_{X} f_{\alpha} d \mu & =\lim _{\alpha} \sum_{\imath=1}^{n} \int_{X} f_{\alpha} \chi_{E_{i}} d \mu \\
& =\left.\sum_{\imath=1}^{n} \lim _{\alpha} \int_{E_{\imath}} f_{\alpha}\right|_{E_{i}} d \mu_{E_{\imath}} \\
& =\sum_{\imath=1}^{n} a_{i} \mu\left(E_{\imath}\right) \\
& =\int_{\lambda} f d \mu .
\end{aligned}
$$

Next we prove the general case. If we put

$$
g_{n}=\sum_{k=1}^{n \cdot 2 n} \frac{k-1}{2^{n}} \chi\left\{x ; \frac{k-1}{2^{n}}<f(x) \leqq \frac{k}{2^{n}}\right\}+n \chi_{\{x, f(x)>n\}},
$$

then we have

$$
\lim _{n} \int_{X} g_{n} d \mu=\int_{X} f d \mu
$$

If we remark that $g_{n}$ is lower semi-continuous, we have

$$
\begin{aligned}
\int_{X} f d \mu & =\lim _{n} \int_{X} g_{n} d \mu=\lim _{n} \lim _{\alpha} \int_{X} f_{\alpha} \wedge g_{n} d \mu \\
& =\lim _{\alpha} \lim _{n} \int_{X} f_{\alpha} \wedge g_{n} d \mu
\end{aligned}
$$




$$
\begin{aligned}
& =\lim _{\alpha} \int_{X} \lim _{n} f_{\alpha} \wedge g_{n} d \mu \\
& =\lim _{\alpha} \int_{X} f_{\alpha} \wedge f d \mu \\
& =\lim _{\alpha} \int_{X} f_{\alpha} d \mu .
\end{aligned}
$$

The lemma is proved.

Lemma 9.5. Let $\mu, \nu$ be $\sigma$-finite pre-Radon measures on topological spaces $X$, $Y$ respectively and $\overline{\mu \otimes \nu}$ be the pre-Radon extension of $\mu \otimes \nu$. Then for every open subset $W$ of $X \times Y$, we have

$$
\overline{\mu \otimes \nu}(W)=\mu_{x} \nu_{y}\left(\chi_{W}(x, y)\right) .
$$

Proof. Let $U$ be the paving generated by

$$
\{U \times V ; U(\text { resp. } V) \text { is open in } X(\text { resp. } Y)\}
$$

and $\left\{W_{\alpha}\right\}$ be a net in $U$ increasing to $W$. By the way similar to Bourbaki [2, $\S 2, n^{\circ} 6$, Prop. 11$]$ the function

$$
x \longmapsto \nu_{y}\left(\chi_{W_{\alpha}}(x, y)\right)
$$

is lower semi-continuous on $X$. By Lemma 9.4 it follows that

$$
\lim _{\alpha} \nu_{y}\left(\chi_{W_{\alpha}}(x, y)\right)=\nu_{y}\left(\chi_{W}(x, y)\right),
$$

which shows that the function

$$
x \longmapsto \nu_{y}\left(\chi_{W}(x, y)\right)
$$

is lower semi-continuous. Hence we have

The proof is complete.

$$
\begin{aligned}
\overline{\mu \otimes \nu}(W) & =\lim _{\alpha} \mu \otimes \nu\left(W_{\alpha}\right) \\
& =\lim _{\alpha} \mu_{x} \nu_{y}\left(\chi_{W}(x, y)\right) \\
& =\mu_{x} \nu_{y}\left(\chi_{W}(x, y)\right) .
\end{aligned}
$$

Under the above preparations, we present a Fubini type theorem.

THEOREM 9.6. Let $\mu, \nu$ be $\sigma$-finte pre-Radon measures on topological spaces $X, Y$ respectuvely and $\overline{\mu \otimes \nu}$ be the pre-Radon extension of $\mu \otimes \nu$. Then for every Borel subset $B$ of $X \times Y$, we have

$$
\overline{\mu \otimes \nu}(B)=\mu_{x} \nu_{y}\left(\chi_{B}(x, y)\right) .
$$

Proof. We recall that for every Borel set $B, \chi_{B}(x, y)$ is separately Borel 
measurable function on $X \times Y$. Let $\mathscr{M}_{1}$ be the class $\left\{E \subset \mathscr{B}(X \times Y) ; \nu_{y}\left(\chi_{E}(x, y)\right)\right.$ is $\mathscr{B}(X)$-measurable\}. Since $\nu$ is $\sigma$-finite, $\mathscr{M}_{1}$ is a monotone class. Furthermore we can easily prove that $\mathscr{H}_{1}$ contains the algebra generated by all open subsets of $X \times Y$. Thus $\mathscr{M}_{1}$ equals $\mathscr{B}(X \times Y)$.

Let $\mathscr{M}_{2}$ be the class

$$
\left\{E \subset \mathcal{B}(X \times Y) ; \overline{\mu \otimes \nu}(E)=\mu_{x} \nu_{y}\left(\chi_{E}(x, y)\right)\right\} .
$$

Since $\mu$ and $\nu$ are $\sigma$-finite, $\mathscr{M}_{2}$ is a monotone class. By Lemma $9.5, \mathscr{M}_{2}$ includes the algebra generated by all open subsets of $X \times Y$. Thus $\mathscr{M}_{2}$ is equal to $\mathscr{B}(X \times Y)$. This completes the proof.

COROLlary 9.7. Let $f$ be a non-negative, extended real valued Borel measurable function on $X \times Y$, then we have

1) $x \longmapsto \int_{Y} f(x, y) d \nu(y)$ is $\mathscr{B}(X)$-measurable ;

2) $y \longmapsto \int_{Y} f(x, y) d \mu(x)$ is $\mathscr{B}(Y)$-measurable ;

3) $\int_{X} d \mu(x) \int_{Y} f(x, y) d \nu(y)=\int_{Y} d \nu(y) \int_{X} f(x, y) d \mu(x)$

$$
=\int_{X \times Y} f(x, y) \overline{d \mu \otimes \nu} \text {. }
$$

Next we consider the countable product of pre-Radon measures.

THEOREM 9.8. Let $\mu_{n}$ be a pre-Radon probability measure on a regular space $X_{n}(n=1,2, \cdots)$. Then the product measure $\mu=\bigotimes_{n=1}^{\infty} \mu_{n}$ on $\left(\prod_{n=1}^{\infty} X_{n}, \bigotimes_{n=1}^{\infty} \mathcal{B}\left(X_{n}\right)\right)$ is uniquely extensible to a pre-Radon measure on the product space $\prod_{n=1}^{\infty} X_{n}$.

Proof. Let $\mathcal{U}$ be the paving generated by

$$
\mathcal{U}_{0}=\left\{\prod_{n=1}^{\infty} U_{n} ; U_{n} \text { is open in } X_{n}, U_{n}=X_{n} \text { except finitely many } n\right\}
$$

and $\left\{W_{\alpha}\right\}$ be a net in $U$ increasing to $\prod_{n=1}^{\infty} X_{n}$. If $\left\{\prod_{n=1}^{\infty} U_{n}^{r}\right\}$ is the collection of open subsets in $U_{0}$ such that each $\prod_{n=1}^{\infty} U_{n}^{r}$ is contained in $W_{\alpha}$ for some $\alpha$, we have $\bigcup_{\gamma} \prod_{n=1}^{\infty} U_{n}^{r}=\prod_{n=1}^{\infty} X_{n}$. We put

$$
\Gamma(k)=\left\{\gamma ; U_{n}^{r}=X_{n} \text { for all } n>k\right\}
$$

and put

$$
U(k)=\bigcup_{\gamma \in \Gamma(k)} \prod_{n=1}^{\infty} U_{n}^{r}
$$

We define a finitely additive set function $\nu$ on the algebra $\mathcal{A}=\bigcup_{n=1}^{\infty}\left(\mathscr{B}\left(\prod_{\imath=1}^{n} X_{\imath}\right)\right.$ 
$\left.\times \prod_{p>n} X_{p}\right)$ as follows :

$$
\nu\left(B_{n} \times \prod_{p>n} X_{p}\right)=\bigotimes_{\imath=1}^{n} \mu_{i}\left(B_{n}\right)
$$

for every $B_{n}$ in $\mathscr{B}\left(\prod_{i=1}^{n} X_{\imath}\right)$, where $\overline{\bigotimes_{i=1}^{n} \mu_{\imath}}$ denotes the pre-Rodon extension of $\bigotimes_{i=1}^{n} \mu_{\imath}$. From Corollary $7.2 \nu$ is well-defined. We shall show $\nu$ is countably additive by the way similar to Halmos $\left[5, \S 38\right.$, Theorem B]. Let $\left\{E_{k}\right\}$ be a decreasing sequence in $\mathcal{A}$. Suppose there exists $\varepsilon>0$ such that $\nu\left(E_{k}\right) \geqq \varepsilon$ for every $k$. We put for every $E=B_{N(E)} \times \prod_{P>N(E)} X_{p}$ in $\mathcal{A}$

$$
E\left(x_{1}, \cdots, x_{n}\right)=\left\{\left(x_{n+1}, x_{n+2}, \cdots\right) ;\left(x_{1}, \cdots, x_{n}, x_{n+1}, \cdots\right) \in E\right\} \text {, }
$$

and

$$
\mu^{(n)}\left(E\left(x_{1}, \cdots, x_{n}\right)\right)=\bar{\bigotimes}_{\imath=n+1}^{\overline{N(E)}} \mu_{i}\left(B_{N(E)}\left(x_{1}, \cdots, x_{n}\right)\right) .
$$

If we put

$$
F_{k}=\left\{x_{1} \in X_{1} ; \mu^{(1)}\left(E_{k}\left(x_{1}\right)\right) \geqq \frac{\varepsilon}{2}\right\} .
$$

then it follows by Theorem 9.6

$$
\begin{aligned}
\nu\left(E_{k}\right) & =\overline{\bigotimes_{i=1}^{\overline{N\left(E_{k}\right)}} \mu_{i}\left(E_{k}\right)} \\
& =\overline{\mu_{1} \otimes\left(\bigotimes_{\imath=2}^{N\left(E_{k}\right)} \mu_{\imath}\right)\left(E_{k}\right)} \\
& =\int_{x_{1}} \mu^{(1)}\left(E_{k}\left(x_{1}\right)\right) d \mu_{1}\left(x_{1}\right) \\
& =\int_{F_{k}} \mu^{(1)}\left(E_{k}\left(x_{1}\right)\right) d \mu_{1}\left(x_{1}\right)+\int_{F_{k}^{c}} \mu^{(1)}\left(E_{k}\left(x_{1}\right)\right) d \mu_{1}\left(x_{1}\right) \\
& \leqq \mu_{1}\left(F_{k}\right)+\frac{\varepsilon}{2} .
\end{aligned}
$$

Thus we have

$$
\mu_{1}\left(F_{k}\right) \geqq \frac{\varepsilon}{2},
$$

which implies there exists $\bar{x}_{1}$ in $X_{1}$ such that

$$
\mu^{(1)}\left(E_{k}\left(\bar{x}_{1}\right)\right) \geqq \frac{\varepsilon}{2}
$$

for every $k$. Similarly there exists $\left(\bar{x}_{n}\right)$ in $\prod_{n=1}^{\infty} X_{n}$ such that 


$$
\mu^{(n)}\left(E_{k}\left(\bar{x}_{1}, \cdots, \bar{x}_{n}\right)\right) \geqq \frac{\varepsilon}{2^{n}}
$$

for every $k$. If we remark that $\left(\bar{x}_{n}\right)$ is in $\bigcap_{k=1}^{\infty} E_{k}, \nu$ is countably additive.

Since $\nu$ is countably additive, for arbitrary $\varepsilon>0$ there exists $k_{0}$ such that

$$
1-\varepsilon<\nu\left(U\left(k_{0}\right)\right)=\bigotimes_{i=1}^{\overline{k_{0}}} \mu_{i}\left(\bigcup_{\gamma \in \Gamma\left(k_{0}\right)}^{k_{0}} \prod_{i=1}^{k_{0}} U_{i}^{\gamma}\right) \text {. }
$$

By Theorem 9.2 there exists $\left\{\gamma_{1}, \cdots, \gamma_{p}\right\}$ in $\Gamma\left(k_{0}\right)$ such that

$$
\bigotimes_{i=1}^{k_{0}} \mu_{i}\left(\bigcup_{q=1}^{p} \prod_{i=1}^{k_{0}} U_{i}^{r_{q}}\right)>1-\varepsilon
$$

Since $\left\{W_{\alpha}\right\}$ is directed, there exists $\alpha_{0}$ such that $W_{\alpha_{0}}$ contains $\bigcup_{q=1}^{p} \prod_{i=1}^{k_{0}} U_{i}^{\gamma_{q}} \times \prod_{m>k_{0}} X_{m}$,

$$
\lim _{\alpha} \mu\left(W_{\alpha}\right)=1 .
$$

By Lemma 9.1 and Theorem 3.2, the restriction $\mu_{0}$ of $\mu$ to $R[\vartheta]=A[U]$ is uniquely extended to a pre-Radon measure $\bar{\mu}$ on $\prod_{n=1}^{\infty} X_{n}$. By Halmos $[5, \S 13$, Theorem A], $\mu$ is identical to $\bar{\mu}$ on the product $\sigma$-algebra $\bigotimes_{n=1}^{\infty} \mathscr{B}\left(X_{n}\right)$. Thus the theorem is proved.

Now we discuss the uncountable product of pre-Radon measures.

THEOREM 9.9. Let $\mu_{\lambda}$ be a pre-Radon probability measure on a regular space $X_{\lambda}(\lambda \in \Lambda)$. Then the product measure $\mu=\otimes_{\lambda \in \Lambda} \xi_{\lambda}$ on $\left(\prod_{\lambda \in \Lambda} X_{\lambda}, \underset{\lambda \in \Lambda}{\otimes} \mathscr{B}\left(X_{\lambda}\right)\right)$ is extended to a unique pre-Radon measure on $\prod_{\lambda \in \Lambda} X_{\lambda}$,

Proof. Let $U$ be the paving generated by

$$
\left\{\prod_{\lambda \in \Lambda} U_{\lambda} ; U_{\lambda} \text { is open in } X_{\lambda}, U_{\lambda}=X_{\lambda} \text { except finitely many } \lambda\right\}
$$

and $\left\{W_{\alpha}\right\}$ be a net in $\mathcal{U}$ increasing to $\prod_{\lambda \in \Lambda} X_{\lambda}$. We put $c=\sup _{\alpha} \mu\left(W_{\alpha}\right)$. Then there exists $\left\{\alpha_{n}\right\}$ such that $c=\lim _{n} \mu\left(W_{\alpha_{n}}\right)=\mu\left(\bigcup_{n=1}^{\infty} W_{\alpha_{n}}\right)$. For simplicity we set $W$ $=\bigcup_{n=1}^{\infty} W_{\alpha_{n}}$. We can write $W_{\alpha}=\bigcup_{n=1}^{N(\alpha)} \prod_{\lambda \in \Lambda} U_{\lambda}^{\alpha, 2}$. If we set

$$
\Lambda_{0}=\left\{\lambda \in \Lambda ; U_{n}^{\alpha_{n}, \imath}=X_{\lambda} \text { for every } n \text { and } \imath=1,2, \cdots, N\left(\alpha_{n}\right)\right\},
$$

then $\Lambda_{1}=\Lambda-\Lambda_{0}$ is a countable set. Since $W$ equals $q_{1}(W) \times \prod_{\lambda \in \Lambda_{0}} X_{\lambda}$, we have $\mu(W)=\left(\otimes_{\lambda \in \Lambda_{1}} \mu_{\lambda}\right)\left(q_{1}(W)\right)$, where $q_{1}$ is the projection of $\prod_{\lambda=\Lambda} X_{\lambda}$ onto $\prod_{\lambda \in \Lambda_{1}} X_{\lambda}$.

In the first step, we assume supp $\mu_{\lambda}$ is equal to $X_{\lambda}$ for every $\lambda$ in $\Lambda$. Sup- 
pose $c<1$. Then there exists $\alpha_{0}$ such that $\left(\otimes_{\lambda \in \Lambda_{1}} \mu_{\lambda}\right)\left(q_{1}\left(W_{\alpha_{0}}\right)-q_{1}(W)\right)>0$, for by Theorem 9.8 it holds $\sup \left(\otimes_{\lambda \in \Lambda_{1}} \mu_{\lambda}\right)\left(q_{1}\left(W_{\alpha}\right)\right)=1$. Therefore, for some $\imath, 1 \leqq \imath \leqq N\left(\alpha_{0}\right)$ it follows $\left(\otimes_{\lambda \in \Lambda_{1}} \mu_{\lambda}\right)\left(\prod_{\lambda \in \Lambda_{1}} U_{\lambda}^{\alpha_{0}, \imath}-q_{1}(W)\right)>0$. If we remark that $\left(\otimes_{\lambda \in \Lambda_{0}} \mu_{\lambda}\right)\left(\prod_{\lambda \in \Lambda_{0}} U_{\lambda}^{\alpha_{0}, i}\right)>0$, we have

$$
\begin{aligned}
c & =\mu\left(W \cup W_{\alpha_{0}}\right) \geqq \mu\left(W \cup \prod_{\lambda \in \Lambda} U_{\lambda}^{\alpha_{0}, i}\right) \\
& =\mu(W)+\mu\left(\prod_{\lambda \in \Lambda} U_{\lambda}^{\alpha_{0}, \imath}-W\right) \\
& =\mu(W)+\mu\left(\left(\prod_{\lambda \in \Lambda_{1}} U_{\lambda}^{\alpha_{0}, \imath}-q_{1}(W)\right) \times \prod_{\lambda \in \Lambda_{0}} U_{\lambda}^{\alpha_{0}, i}\right) \\
& =\mu(W)+\left(\otimes_{\lambda \in \Lambda_{1}} \mu_{\lambda}\right)\left(\prod_{\lambda \in \Lambda_{1}} U_{\lambda}^{\alpha_{0}, \imath}-q_{1}(W)\right)\left(\bigotimes_{\lambda \in \Lambda_{0}} \mu_{\lambda}\right)\left(\prod_{\lambda \in \Lambda_{0}} U_{\lambda}^{\alpha_{0}, i}\right) \\
>c, &
\end{aligned}
$$

which is a contradiction. Hence we obtain $\mu(W)=1$. If follows that

$$
\lim _{\alpha} \mu\left(W_{\alpha}\right)=1=\mu\left(\prod_{\lambda \in \Lambda} X_{\lambda}\right) .
$$

We shall prove the general case. By $\nu_{\lambda}$ we denote the restriction of $\mu_{\lambda}$ to $Y_{\lambda}=\operatorname{supp} \mu_{\lambda}$. Since the net $\left\{W_{\alpha} \bigcap_{\lambda \in \Lambda} Y_{\lambda}\right\}$ increases to $\prod_{\lambda \in \Lambda} Y_{\lambda}$, from the firs: step we have

$$
\begin{aligned}
\lim _{\alpha}\left(\otimes_{\lambda \in \Lambda} \mu_{\lambda}\right)\left(W_{\alpha}\right) & =\lim _{\alpha}\left(\otimes_{\lambda \in \Lambda} \nu_{\lambda}\right)\left(W_{\alpha} \cap \prod_{\lambda \in \Lambda} Y_{\lambda}\right) \\
& =\left(\otimes_{\lambda \in \Lambda} \nu_{\lambda}\right)\left(\prod_{\lambda \in \Lambda} Y_{\lambda}\right) \\
& =\left(\otimes_{\lambda \in \Lambda} \mu_{\lambda}\right)\left(\prod_{\lambda \in \Lambda} X_{\lambda}\right) .
\end{aligned}
$$

By Lemma 8.1 and Theorem 3.2, the restriction $\mu_{0}$ of $\mu$ to $R[U]=A[V]$ is uniquely extensible to a pre-Radon measure $\bar{\mu}$ on $\prod_{\lambda \in A} X_{\lambda}$. By Halmos $[5, \S 13$, Theorem A], $\mu$ is identical with $\bar{\mu}$ on the product $\sigma$-algebra $\bigotimes_{\lambda \in \Lambda} \mathscr{B}\left(X_{\lambda}\right)$. This proves Theorem 9.9. 9.9 .

We denote by $\overline{\bigotimes_{\lambda \in \Lambda} \mu_{\lambda}}$ the pre-Radon extension of $\otimes_{\lambda \in \Lambda} \mu_{\lambda}$ obtained in Theorem

Lastly we consider the product measure of Radon measures. The following lemma is well-known (for example see Bourbaki [2, $\$ 4$, Théorème 2]).

Lemma 9.10. Let $\mu_{n}$ be a Radon probability measure on a regular space $X_{n}$ $(n=1,2, \cdots)$. Tnen the pre-Radon extension $\overline{\bigotimes_{n=1}^{\infty} \mu_{n}}$ of $\bigotimes_{n=1}^{\infty} \mu_{n}$ is a Radon measure on $\prod_{n=1}^{\infty} X_{n}$. 
THEOREM 9.11. Let $\mu_{\lambda}$ be a Radon probability measure on a regular space $X_{\lambda}(\lambda \in \Lambda)$. Then the pre-Radon extension $\overline{\bigotimes_{\lambda \in \Lambda} \mu_{\lambda}}$ is a Radon measure if and only if $\operatorname{supp} \mu_{\lambda}$ is a compact subset of $X_{\lambda}$ except countably many $\lambda$ in $\Lambda$.

Proof. Let $\otimes_{\lambda \in \Lambda} \mu_{\lambda}$ be a Radon measure. Suppose that there exists some uncountable subset $\Lambda_{0}$ of $\Lambda$ such that supp $\mu_{\lambda}$ is not compact for every $\lambda$ in $\Lambda_{0}$. Without loss of generality we may assume $\Lambda_{0}$ is equal to $\Lambda$. For each compact subset $K$ of $\prod_{\lambda \in \Lambda} X_{\lambda}$, we shall show $\overline{\bigotimes_{\lambda \in \Lambda} \mu_{\lambda}}(K)=0$. Putting

$$
\Lambda_{n}=\left\{\lambda \in \Lambda ; \mu_{\lambda}\left(p_{\lambda}(K)\right)<1-\frac{1}{n+1}\right\}
$$

we have $\Lambda=\bigcup_{n=1}^{\infty} \Lambda_{n}$, where $p_{\lambda}$ is the projection of $\prod_{\lambda \in \Lambda} X_{\lambda}$ onto $X_{\lambda}$. Since $\Lambda$ is uncountable, there exists $n$ such that $\Lambda_{n}$ is infinite. Hence $\Lambda_{n}$ has an infinitely countable subset $\left\{\lambda_{\imath} ; \imath=1,2, \cdots\right\}$. Thus we have

$$
\begin{aligned}
\bar{\bigotimes}_{\lambda \in \Lambda} \mu_{\lambda}(K) & \leqq \overline{\bigotimes_{\lambda \in \Lambda} \mu_{\lambda}}\left(\prod_{\lambda \in \Lambda} p_{\lambda}(K)\right) \\
& \leqq \overline{\bigotimes_{\lambda \in \Lambda} \mu_{\lambda}}\left(\prod_{i=1}^{\infty} p_{\lambda_{i}}(K) \times \prod_{\lambda \neq \lambda_{i}} X_{\lambda}\right) \\
& =\lim _{k}\left(\bigotimes_{\lambda \in \Lambda} \mu_{\lambda}\right)\left(\prod_{i=1}^{k} p_{\lambda_{i}}(K) \times \prod_{\lambda \neq \lambda_{2}} X_{\lambda}\right) \\
& =\lim _{k} \mu_{\lambda_{1}}\left(p_{\lambda_{1}}(K)\right) \cdots \mu_{\lambda_{k}}\left(p_{\lambda_{k}}(K)\right) \\
& \leqq \lim _{k}\left(1-\frac{1}{n+1}\right)^{k}=0 .
\end{aligned}
$$

Therefore we have

$$
\overline{\otimes_{\lambda \in \Lambda} \mu_{\lambda}}\left(\prod_{\lambda \in \Lambda} X_{\lambda}\right)=0
$$

This is a contradiction.

The converse follows by Lemma 9.10. Thus we have proved Theorem 9.11.

\section{Appendix}

We shall examine the relation between inner regularity (*) and outer regularity $(* *)$ :

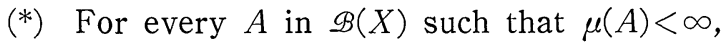

$$
\mu(A)=\operatorname{supp}\{\mu(F) ; F \subset A \text { and } F \text { is closed }\} ;
$$

(**) For every $A$ in $\mathscr{B}(X)$, 


$$
\mu(A)=\inf \{\mu(O) ; O \supset A \text { and } O \text { is open }\} .
$$

Theorem A. Let $\mu$ be a Borel measure on a topological space $X$ such that $X=\bigcup_{n=1}^{\infty} U_{n}$ and $\mu\left(U_{n}\right)$ is finte for countable open subsets $\left\{U_{n}\right\}$. Then $(*)$ implies $(* *)$.

Proof. Let $A$ be a Borel subset. Then for every positive $\varepsilon$, there exists a closed subset $F_{n}$ contained in $A^{c} \cap U_{n}$ such that $\mu\left(A^{c} \cap U_{n}-F_{n}\right)<\varepsilon / 2^{n}$. If we put $V_{n}=U_{n} \cap F_{n}^{c}$, then we have

$$
\begin{aligned}
\mu\left(\bigcup_{n=1}^{\infty} V_{n}-A\right) & \leqq \mu\left(\bigcup_{n=1}^{\infty} V_{n}-\bigcup_{n=1}^{\infty}\left(U_{n} \cap A\right)\right) \\
& \leqq \sum_{n=1}^{\infty} \mu\left(V_{n}-U_{n} \cap A\right) \\
& <\varepsilon
\end{aligned}
$$

This completes the proof.

COROLlary. Let be a $\sigma$-compact locally compact space and $\mu$ be a Borel measure on $X$ such that $\mu(K)$ is finite for every compact set $K$. Then (*) implies (**).

Theorem B. Let $\mu$ be a Borel measure on a topological space X. If $\mu$ is $\sigma$ finte and satısfies (**), then (*) holds.

Proof. Let $E_{n}$ be a Borel subset of finite measure such that $X=\bigcup_{n=1}^{\infty} E_{n}$. For every $A$ in $\mathscr{B}(X)$ and every positive $\varepsilon$, there exists and open subset $U_{n}$ containing $A^{c} \cap E_{n}$ such that $\mu\left(U_{n}-A^{c} \cap E_{n}\right)<\varepsilon / 2^{n}$. Then we have

$$
\begin{aligned}
\mu(A)-\mu\left(\bigcap_{n=1}^{\infty} U_{n}^{c}\right) & =\mu\left(A \cap\left(\bigcup_{n=1}^{\infty} U_{n}\right)\right) \\
& =\mu\left(\bigcup_{n=1}^{\infty} U_{n}-\bigcup_{n=1}^{\infty}\left(A^{c} \cap E_{n}\right)\right) \\
& \leqq \sum_{n=1}^{\infty} \mu\left(U_{n}-A^{c} \cap E_{n}\right) \\
& <\varepsilon,
\end{aligned}
$$

which proves the theorem.

Lastly according to the comments of Fremlin we note the relation between pre-Radon measures and quasi-Radon measures.

1) Let $\mu$ be a pre-Radon measure on a topological space $X$. Then there exists a unique quasi-Radon measure $\nu$ on $\left(X, \Sigma_{\mu^{*}}\right)$ such that for any $B$ in $\Sigma_{\mu^{*}}$ with $\mu^{*}(B)<\infty, \nu(B)=\mu^{*}(B)$, where $\mu^{*}$ is the outer measure derived from $\mu$ and $\Sigma_{\mu^{*}}$ is the family of all $\mu^{*}$-measurable sets. Moreover it holds that $\nu(O)=\mu(O)$ 
for every open set $O$.

2) Let $\nu$ be a locally finite quasi-Radon measure on $(X, \Sigma)$. Then there uniquely exists a pre-Radon measure $\mu$ on $X$ such that $\mu(O)=\nu(O)$ for every open set $O$.

\section{REFERENCES}

[1] Amemiya, I., On measures on topological spaces, Kōkyu-roku of R.I.M.S., Kyoto Univ., No. 96 (1970), 73-80 (in Japanese).

[2] Bourbaki, N., Intégration, Chapitre 9, Hermann (1969).

[3] Fremlin, D. H., Topological Riesz spaces and measure theory, Cambridge Univ. Press (1973).

[4] Gardner, R. J., The regularity of Borel measures and Borel measure-compactness, Proc. London Math. Soc. (3) 39 (1975), 90-113.

[5] Halmos, P. R., Measure theory, Van Nostrand (1950).

[6] Hewitt, E. Ahd Ross, K.A., Abstract harmonic analysis I, Springer-Verlag (1963).

[7] Hewitt, E. And Stromberg, K., Real and abstract analysis, Springer-Verlag(1965).

[8] Hirschfeld, R. A., Riots, Nieuw Archief voor Wiskunde (3) 22 (1974), 1-43.

[9] KIRK, R. B., Locally compact, B-compact spaces, Indag. Math. 31 (1969), 333-344.

[10] KirK, R. B. ANd Crenshaw, J.A., A generalized topological measure theory, Trans. Amer. Math. Soc. 207 (1975), 189-217.

[11] Schwartz, L., Radon measures on arbitrary topological spaces and cylindrical measures, Oxford Univ. Press (1973).

[12] Tortrat, A., Sur les lois $\tau$-reguliers, leures produits et leur convolution, Transaction of the 6th Prague Conference on Information Theory, Statistical Decision Functions, Random Process (1971), 839-849.

[13] Varadarajan, V.S., Measures on topological spaces, Mat. Sbornik 55 (97) (1961), 33-100 (Russian); American Math. Soc. Translations, Series 2, 48. (1965), 161-228.

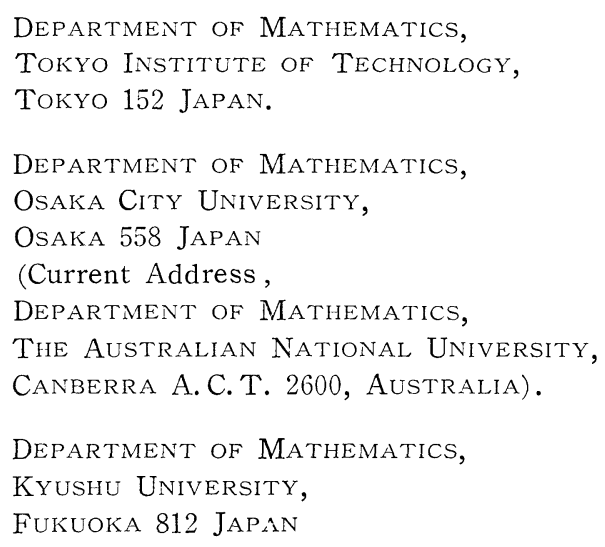

